\title{
Using Jacobi iterations and blocking for solving sparse triangular systems in incomplete factorization preconditioning
}

Article

Accepted Version

Creative Commons: Attribution-Noncommercial-No Derivative Works 4.0

Chow, E., Hartwig, A., Scott, J. and Dongarra, J. (2018) Using Jacobi iterations and blocking for solving sparse triangular systems in incomplete factorization preconditioning. Journal of Parallel and Distributed Computing, 119. pp. 219-230. ISSN 0743-7315 doi: https://doi.org/10.1016/j.jpdc.2018.04.017 Available at https://centaur.reading.ac.uk/77054/

It is advisable to refer to the publisher's version if you intend to cite from the work. See Guidance on citing.

To link to this article DOI: http://dx.doi.org/10.1016/j.jpdc.2018.04.017

Publisher: Elsevier

All outputs in CentAUR are protected by Intellectual Property Rights law, including copyright law. Copyright and IPR is retained by the creators or other copyright holders. Terms and conditions for use of this material are defined in the End User Agreement.

www.reading.ac.uk/centaur 
Central Archive at the University of Reading

Reading's research outputs online 


\title{
Using Jacobi Iterations and Blocking for Solving Sparse Triangular Systems in Incomplete Factorization Preconditioning
}

\author{
Edmond Chow ${ }^{\mathrm{a}, *}$, Hartwig Anzt ${ }^{\mathrm{b}, \mathrm{c}}$, Jennifer Scott ${ }^{\mathrm{d}}$, Jack Dongarra ${ }^{\mathrm{c}, \mathrm{e}, \mathrm{f}}$ \\ ${ }^{a}$ School of Computational Science and Engineering, Georgia Institute of Technology, USA \\ ${ }^{b}$ Steinbuch Centre for Computing, Karlsruhe Institute of Technology, Germany \\ ${ }^{c}$ Innovative Computing Laboratory, University of Tennessee, USA \\ ${ }^{d}$ Scientific Computing Department, STFC Rutherford Appleton Laboratory, UK \\ ${ }^{e}$ School of Mathematics and School of Computer Science, University of Manchester, UK \\ ${ }^{f}$ Oak Ridge National Laboratory, USA
}

\begin{abstract}
When using incomplete factorization preconditioners with an iterative method to solve large sparse linear systems, each application of the preconditioner involves solving two sparse triangular systems. These triangular systems are challenging to solve efficiently on computers with high levels of concurrency. On such computers, it has recently been proposed to use Jacobi iterations, which are highly parallel, to approximately solve the triangular systems from incomplete factorizations. The effectiveness of this approach, however, is problem-dependent: the Jacobi iterations may not always converge quickly enough for all problems. Thus, as a necessary and important step to evaluate this approach, we experimentally test the approach on a large number of realistic symmetric positive definite problems. We also show that by using block Jacobi iterations, we can extend the range of problems for which such an approach can be effective. For block Jacobi iterations, it is essential for the blocking to be cognizant of the matrix structure.

Keywords: sparse linear systems, triangular solves, iterative solvers, preconditioning.
\end{abstract}

\section{Introduction}

As computers increasingly rely on various forms of parallelism to obtain high performance, the design and performance optimization of large-scale numerical algorithms must focus on the efficient exploitation of parallel architectures. Unfortunately, 5 simple algorithms frequently become remarkably complex when implemented in parallel and the most efficient parallel algorithm may not be the one that is most intuitive.

In this paper, we investigate a non-intuitive approach to solving the sparse triangular systems that arise when using incomplete factorization preconditioners with an

\footnotetext{
${ }^{*}$ Corresponding author

Email addresses: echow@cc.gatech.edu (Edmond Chow), hanzt@icl.utk.edu (Hartwig Anzt), jennifer.scott@stfc.ac.uk (Jennifer Scott), dongarra@icl.utk.edu (Jack Dongarra)
} 
iterative method for solving sparse linear systems. The conventional method of solving triangular systems is to use forward or backward substitution and this can be parallelized using level scheduling $[1,2,3,4,5]$. Here we investigate using an iterative method-in particular, Jacobi relaxation. The use of an iterative method is feasible when an approximate solution is acceptable, as in the case of preconditioning. The approach is less applicable to the case of solving with factors from a direct (not incom-

15 plete) factorization where the factors are much less sparse and typically an exact solve is desired.

In recent previous work, this approach demonstrated significant reductions in total solution time for the preconditioned conjugate gradient (PCG) method on highly parallel architectures such as Intel Xeon Phi co-processors and graphics processing units 20 (GPUs) [6, 7], even though additional PCG iterations may be required to achieve the requested accuracy. The improved speed for each triangular solve is because, for some problems, particularly with high levels of fill in the triangular factors, level scheduling is unable to reveal sufficient parallelism to fully exploit the GPU hardware. On the other hand, Jacobi relaxation, which primarily relies on the sparse matrix vector prod25 uct (SpMV) operation to compute a residual vector, is highly parallel and can exploit the substantial efforts that have been invested in optimizing SpMV on various parallel architectures.

Although the iterative triangular solve approach can result in significant speedups on highly parallel architectures, the approach does not work on all problems. It is posso sible for the iterations on the triangular systems to converge too slowly and thus be uncompetitive with the conventional level-scheduled approach. The goal of this paper is to investigate the range of applicability of using iterative triangular solves for incomplete factorization preconditioning by testing it with a large set of sparse symmetric positive definite linear systems. Further, we introduce the idea of using block Jacobi ${ }^{5}$ iterations, ${ }^{1}$ which improves the robustness of the iterative approach. Our hypothesis is that for matrices that model many types of physical systems, especially partial differential equations, an iterative approach to solving the systems involving its triangular factors can be effective. In particular, although these matrices may not be diagonally dominant and may be ill-conditioned, many have relatively large diagonal entries com-

40 pared to the off-diagonal entries. If the incomplete factorizations of these matrices are stable, they are also likely to have relatively large diagonal entries. Systems with such matrices can typically be solved efficiently by iterative methods. If convergence is poor, a block diagonal scaling can improve diagonal dominance.

Alternatives to sparse triangular solves for incomplete factorization preconditioning have been proposed before. One major alternative is to compute and use sparse approximate inverses of the incomplete factors, so that preconditioning reduces to SpMV operations $[8,9]$. Related to this is representing the inverse of a sparse triangular matrix as the product of sparse triangular factors $[10,11]$. Another possible approach is to use Neumann series approximations to the inverse of the incomplete factors [8, 12].

50 Preconditioning is again reduced to a sequence of SpMV operations. This approach

\footnotetext{
${ }^{1}$ Initial ideas were presented orally at the 2015 SIAM Conference on Computational Science and Engineering.
} 
was found to be potentially competitive with other techniques for nonsymmetric problems but it lacks robustness, while for symmetric problems a number of more efficient alternatives are available [8]. The Neumann series technique is the same as the Jacobi relaxation approach investigated here if a diagonal scaling is first applied to the system in the former. Recently, based on the encouraging results in [7] for using Jacobi iterations to solve triangular systems, Huckle and coauthors [13] have suggested the use of stationary iterations for solving sparse triangular systems based on sparse approximate inverses.

In the symmetric case, the amount of parallelism in level-scheduled sparse triangular solves can be increased by using multicolor reordering of the rows and columns of the original matrix and computing the incomplete Cholesky factors of this reordered matrix [14]. However, multicolor reorderings generally give poorer PCG convergence results compared to other orderings such as the band reducing RCM ordering [15] or profile reducing Sloan ordering [16] (see, for example, [17, 18, 19, 20, 21, 22]). For some "easy" problems, the convergence rate may be degraded by as much as 60 to $100 \%$ but this can be compensated for by the additional parallelism in the solves. For harder problems, however, multicolor reorderings may result in no convergence.

The rest of this paper is organized as follows. In Section 2, we describe the use of Jacobi and block Jacobi relaxation for the iterative solution of triangular systems. In 70 particular, various blocking strategies are reviewed. Section 3 presents our experimental study and demonstrates the potential effectiveness of Jacobi and block Jacobi solves using a large set of test problems. Concluding remarks are made in Section 4.

\section{Background and Methodology}

\subsection{Jacobi and block Jacobi relaxation for triangular systems}

Consider a triangular system of equations

$$
R y=c,
$$

where $R$ is either upper or lower triangular. In our application in which we want to solve the system $A x=b, R$ is an incomplete factor of $A$ and, in particular, in the symmetric case, $R=L$ or $L^{T}$, where $L$ is an incomplete Cholesky factor of $A$. The Jacobi iteration for solving (1) is

$$
y_{k+1}=y_{k}+D^{-1}\left(c-R y_{k}\right),
$$

where $D$ is the diagonal of $R$. For the sparse triangular solves, we take the initial guess

$$
y_{0}=D^{-1} c .
$$

We will refer to the number of Jacobi iterations performed as the number of Jacobi sweeps. The iteration matrix

$$
G=I-D^{-1} R
$$

is strictly triangular with a zero diagonal. Thus the iteration is guaranteed to converge. However, in practice, because $G$ is triangular and thus non-normal, the iteration may diverge before converging. If this initial divergence stage is long and/or causes numerical 
overflow, then using Jacobi iterations will not be an effective method of solution. Thus effectiveness will depend on the degree of non-normality of $G$. Non-normality can be measured in different ways. For triangular matrices, having only small off-diagonal entries results in a small departure from non-normality.

90 Block Jacobi relaxation or, equivalently, block scaling of $R$, can reduce non-normality. If a block structure is imposed on $R$ then it can be trivially written as the sum

$$
R=\tilde{R}+D
$$

where $\tilde{R}$ is strictly block triangular and $D$ is now block diagonal with $i$-th block $D_{i i}$. The iteration is the same as in (2), where the $i$-th block row of the iteration matrix (4) now has off-diagonal blocks $G_{i j}=D_{i i}^{-1} R_{i j}(j \neq i)$. If the norm of $D_{i i}^{-1}$ is small, then 95 the norm of the off-diagonal blocks will be small, as desired. As a proxy, we will seek to maximize the size of the entries in the diagonal blocks of $A$. This blocking for $A$ is then imposed on the lower and upper triangular incomplete factors.

Observe that, in general, we seek a blocking in which the block size is small compared to the order $n$ of $A$ so as to limit the cost of applying block Jacobi. With small blocks, the cost of block Jacobi is not significantly greater than for scalar Jacobi because the main cost in both is the computation of the residual $c-R y_{k}$. For small blocks, one possibility to assist with efficiency is to store the inverses $D_{i i}^{-1}$ explicitly. We also note that the matrix $D^{-1} R$ is not formed, as this may require more storage than storing $D^{-1}$ and $R$ separately.

\subsection{Blocking vs. reordering}

The most general form of blocking involves two steps: (1) identifying rows (or columns) of a matrix that should be grouped together into a "block," and then (2) reordering the matrix such that the rows within each block are numbered consecutively. (We use the term "block" to mean either a group of rows or columns (or their indices), 110 or a submatrix of a matrix; the meaning should be clear from the context.) For symmetric matrices, the rows and columns are reordered the same way. It is known, however, that reordering a matrix affects the quality of its incomplete factorization, and that some orderings, such as RCM, are better than others when these factorizations are used as preconditioners. Arbitrary blockings (and thus reorderings) of a matrix can lead to a 15 failure of the incomplete factorization [23].

Blocking, however, may not require the first reordering step, and these cases should be distinguished from the general case. For a matrix that already has a desired ordering, it is possible to group consecutively numbered rows (and columns) together. The simplest way to impose such a blocking is to divide the rows into the requested number 120 of blocks such that each block contains (approximately) the same number of rows. A locality-preserving band or profile reducing ordering, such as RCM or Sloan, permutes nonzero entries of the matrix close to the diagonal of the matrix, and thus these entries are likely to lie in the block diagonal part of the reordered matrix.

In this paper, we use a simple blocking technique that we call supervariable amalgamation, which is described in the next subsection. For completeness, we also briefly mention other blocking techniques. 


\subsection{Supervariable blocking and supervariable amalgamation}

The set of variables that correspond to a set of matrix columns with the same sparsity pattern is called a supervariable. Supervariables occur frequently as a result of each node or element of a partial differential equation (PDE) discretization having multiple variables or degrees of freedom associated with it. These variables are typically tightly coupled, leading to dense diagonal blocks with large norm.

If a matrix has a supervariable structure, then it is natural to associate each supervariable with a block. Assuming that the variables in a supervariable are numbered consecutively, then this blocking does not require reordering the matrix. If blocks larger than the size of the supervariables are desired, then larger blocks can be formed by amalgamating supervariables.

When amalgamating adjacent supervariables, it is important that these supervariables represent adjacent or nearby nodes or elements in the PDE mesh. This is because 140 there is no advantage in grouping variables together that are not coupled within the block. To help ensure that adjacent supervariables are coupled, the matrix should be ordered so that variables that are close in the ordering are nearby in the PDE mesh. This can be accomplished by applying a locality-preserving ordering to the supervariable structure (called the condensed graph) before the supervariables are amalgamated.

145 Fortunately, as already observed, locality-preserving orderings such as RCM are also beneficial for incomplete factorization preconditioners.

In this paper, instead of identifying a supervariable structure and then applying $\mathrm{RCM}$ ordering to that structure, we simply apply RCM ordering to the original rows and columns of the matrix before identifying supervariables. RCM ordering tends to 150 preserve any existing supervariable structure because variables belonging to a supervariable will tend to stay numbered together.

The input to the supervariable amalgamation algorithm is the sparsity pattern of a matrix that has been reordered using RCM. The supervariable structure is found by comparing the sparsity patterns of adjacent matrix columns. Supervariables are then amalgamated into blocks with a given maximum block size $m$ by merging adjacent supervariables. The algorithm does not alter the ordering of the matrix and only outputs the grouping of consecutive rows and columns into blocks. We note that when identifying supervariables, if there are supervariable blocks that are larger than the maximum allowed block size, then these blocks are recursively divided until the block size limit is satisfied.

When identifying supervariables, techniques based on hashing are available to rapidly determine if the structure of two columns differ [24, 25] (further discussion of efficient algorithms for identifying supervariables is given in [26]). In some cases, two columns may be associated to different variables at the same PDE grid point, but may not have identical structure because of numerical zero values in the columns. In this case, approximate matching of column structures can be useful, and the "cosine" between the structure of two columns can be checked [25]. In this paper, however, we use exact matching to identify supervariables. (In general, the cosine technique can be used to identify variables that are "near" one another; however, we use the simpler $\mathrm{RCM}$ algorithm for this.) 


\subsection{Other blocking techniques}

Many blocking techniques can be interpreted as graph partitioning. A sparse matrix can be associated with a graph, where the matrix rows or columns (in the symmetric case) are identified with graph nodes, and nonzeros are identified with graph edges. Graph-based approaches can be used to find a blocking of a sparse matrix, where each block corresponds to a partition of the graph nodes. Usually, the partitions of the graph are contiguous, and thus the nodes in the same partition are nearby in the graph. Graph partitioning techniques minimize the number of edges shared between partitions, which corresponds to minimizing the number of nonzeros that fall outside the diagonal the number of nonzero entries outside the diagonal block structure is not explicitly minimized.

Graph-based approaches generally block together matrix rows and columns that are not necessarily numbered together; therefore, reordering the matrix is necessary. However, the ordering of the blocks is not specified by a graph partitioning. If an ordering to produce an accurate incomplete factorization is desired, then it can be effective to order the block structure (the condensed graph) using RCM or the Sloan algorithm, especially if the block sizes are small. The ordering of the variables within blocks is also flexible; one choice is to order the variables within a block in ascending order of heir original numbering

Graph partitioning approaches, e.g., the package METIS [27], typically partition a coarse version of the graph, and then uncoarsen and refine the partitioning over several stages called levels. When large numbers of small partitions are desired (as in our case where the partitions are often so small that the corresponding diagonal blocks are almost dense) then the original graph cannot be coarsened too far, and the quality of the result depends highly on the quality of the partitioning of the coarsest version of the graph.

A different graph-based blocking approach is used by PABLO (parametrized block ordering) $[28,29]$, which is designed for block Jacobi and block Gauss-Seidel preconditioning. Here, blocks are constructed one-by-one. A block initially consists of a single node. Additional nodes are added to the block if they satisfy a "fullness" criterion (maximizing the number of connections between nodes in the same block) or a "connectivity" criterion (minimizing the number of connections between blocks) until a maximum block size is reached.

All the algorithms we have described so far utilize only the structure of the sparse matrix, and not its values. More effective methods may be constructed if values are also considered, explicitly placing large entries in the block diagonal portion of the blocked matrix. METIS can partition graphs with weighted edges by minimizing weighted edge cuts. Edge weights may be defined as being proportional to the absolute values of the corresponding off-diagonal matrix entries. PABLO has been extended to TPABLO (threshold PABLO) and the family of PABLO algorithms is implemented as the package XPABLO [28]. TPABLO uses a threshold on the magnitude of the nonzeros in a matrix when applying its fullness and connectivity criteria. All nonzeros above the threshold are treated equally, while those below the threshold are discounted. A possible disadvantage of TPABLO is that it does not prioritize a large nonzero over a smaller nonzero if they are both above or below the threshold. 
A blocking algorithm sensitive to matrix values that does not use a threshold is the HDPRE algorithm of Duff and Kaya [30]. A block diagonal structure is generated by considering the nonzero values in decreasing order of their size. The HDPRE algorithm is designed for matrices with nonsymmetric structure and utilizes the idea of partitioning a graph such that each subcomponent is strongly connected [31]. For our symmetric positive definite test problems, we use a much simpler version of the algorithm, which we call priority blocking that we describe next.

\subsection{Priority blocking algorithms}

In priority blocking, a block is identified by a set of graph nodes. Initially, each graph node corresponds to a block or set of size 1. Each nonzero or graph edge considered creates a new diagonal block, to which the nonzero will belong, by merging two existing blocks, unless the resulting block exceeds a prescribed maximum size. By considering nonzeros in decreasing order of their size, large nonzeros are effectively placed into the diagonal block structure before small nonzeros.

The priority blocking algorithm is specified as Algorithm 1. For efficiency, a priority queue, implemented as a heap, is used to select the nonzeros in order of decreasing size. The algorithm provides a blocking but does not specify an ordering. As for other graph-based blocking approaches, RCM ordering can be applied to the condensed graph.

The priority blocking algorithm with dynamic edge weights is specified in Algorithm 2. This algorithm attempts to improve the priority blocking algorithm by adjusting the edge weights (absolute value of the corresponding nonzero element) when blocks are merged. Consider edge $(i, j)$ where node $i$ belongs to set $S_{l_{i}}$ and node $j$ belongs to set $S_{l_{j}}$. Also assume that there exists a node $k$ such that edges $(i, k)$ and $(j, k)$ exist in the graph but have yet to be considered by the priority blocking algorithm. When sets $S_{l_{i}}$ and $S_{l_{j}}$ are merged, then the edges $(i, k)$ and $(j, k)$ collapse into a single edge between node $k$ and the newly created block. The new edge replaces the collapsed edges and has weight equal to the sum of the weights of the edges it replaces.

245 This technique is analogous to graph coarsening (see, for example, [27]).

Again, a priority queue, implemented as a heap, is used to store and extract the edges in the graph. When a new edge replaces two or more collapsed edges, one of these edges has its weight increased and the other edges are removed. This entails updating the heap data structure. Although increasing the priority of an item in a heap can be done efficiently, in time proportional to $\log _{2}$ of the number of elements in the heap, not all priority queues implement this operation. In this case, we can simply add the edge with the updated weight to the heap. The extra edges (with smaller weights) will be removed from the heap in turn, but will have no effect on the result of the algorithm. The least scalable component of the priority blocking algorithms is choosing the edge with the largest weight. This cost is $O\left(n_{z} \log _{2} n_{z}\right)$ when a heap is used, where $n_{z}$ is the number of nonzeros in the matrix.

\section{Results and Discussion}

Our goal in this paper is to test the use of iterative methods for approximately solving the triangular systems that arise when an incomplete Cholesky factorization is 


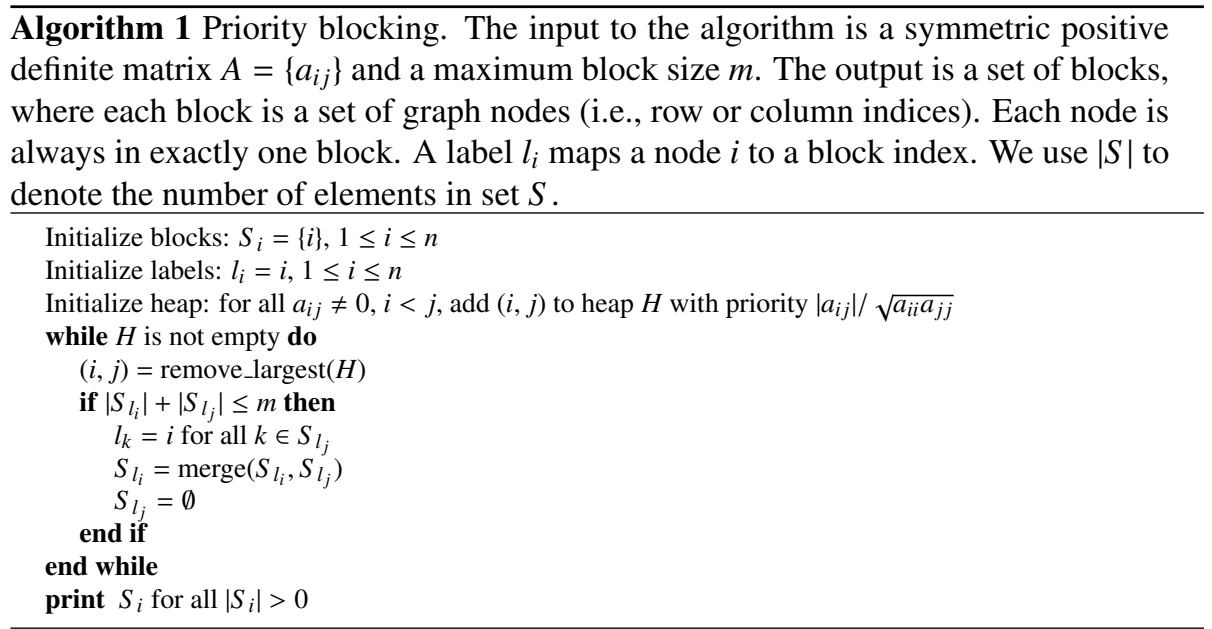

used as a preconditioner in PCG. We are interested in assessing the overall effectiveness of both scalar Jacobi and block Jacobi methods. For the latter, we briefly examine the use of alternative blocking strategies.

In our numerical experiments, we use symmetric positive definite matrices from the SuiteSparse Matrix Collection [32]. For the results reported in this paper, RCM 265 ordering is used unless indicated otherwise. Each test matrix $A$ is scaled on the left and right by a diagonal matrix whose $j$-th diagonal entry is the reciprocal square root of the 2-norm of column $j$ of $A$. We use $\operatorname{IC}(k)(k=0,1)$ to denote an incomplete Cholesky factorization of level $k$; IC $(k)$-PCG denotes PCG with IC $(k)$ preconditioning. For each matrix, we use IC $(k)$-PCG to solve a system of equations where the right-hand side vector is the vector of all ones. The initial approximation to the solution is the zero vector. IC $(k)$-PCG is considered to have converged when the residual 2-norm relative to the initial residual 2-norm is less than $10^{-6}$.

Timings (all reported in seconds) are collected using an NVIDIA Tesla K40 GPU, using double precision arithmetic. This GPU consists of 15 Streaming Multiprocessors, each containing 64 double precision (DP) units. For both the scalar and block Jacobi triangular solves, we map matrix rows to threads. Theoretically, this strategy fills the DP units for matrices with at least 960 rows. However, for efficient processing it is essential to oversubscribe the processing units and switch between contexts to hide main memory latency. In our experiments, we consider matrices with 1000 to 1.5 million rows. The small problems are too small to efficiently utilize the hardware, but we expect that the GPU easily hides the memory latency for the large problems.

The algorithms are implemented using the MAGMA-sparse software package, part of the MAGMA open source software library [33]. MAGMA-sparse uses libraries and compilers from CUDA version 8.0. In particular, exact triangular solves using parallel level-scheduled forward/backward substitution (trsm) use the code implemented in the NVIDIA cuSPARSE library. For the block Jacobi solves, the diagonal blocks are inverted using a batched Gauss-Jordan elimination GPU kernel [34]. We note that we store the inverse of a block diagonal matrix, $D^{-1}$, as a sparse matrix. For higher effi- 


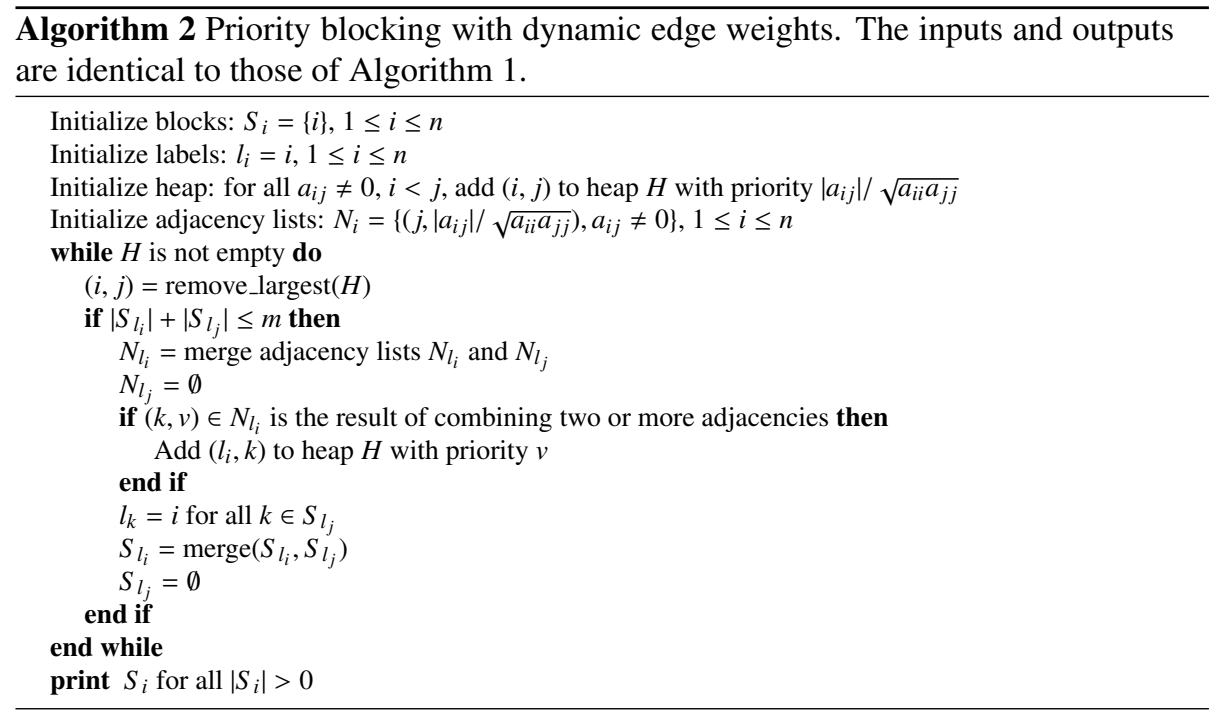

ciency, $D^{-1}$ could be stored using a specialized data structure as a sequence of dense matrices corresponding to its diagonal blocks.

\subsection{Examples}

In Figure 1 we present a detailed analysis for the matrix Hook_1498. Figure 1a shows the residual norm history (relative to the norm of the right-hand side) for a single solve with the lower triangular IC( 0 ) factor of the matrix using the scalar Jacobi method. We use a random right-hand side vector, and zero sweeps corresponds to using the initial guess (3). For solving a system of equations with the Hook_1498 matrix, Figure $1 \mathrm{~b}$ reports the number of IC(0)-PCG iterations required for convergence when up to 6 Jacobi sweeps are used for each triangular solve with the $\mathrm{IC}(0)$ factors. As the number of Jacobi sweeps increases, the number of iterations approaches 1338, which is the number required if exact triangular solves are used, corresponding to the conventional approach.

A single scalar Jacobi sweep on an $\mathrm{IC}(0)$ factor takes 6.5 milliseconds for this problem, which is more than $33 \times$ faster than the cuSPARSE triangular solve, taking 216.7 milliseconds. As we see in Figure 1b, a single Jacobi sweep does not provide sufficient accuracy, and also few sweeps degrade the preconditioner quality making additional PCG iterations necessary. In terms of the PCG run time, the optimal number of Jacobi sweeps for this problem is 3 , see Figure 1c. With 3 sweeps, the residual norm for a triangular solve is reduced by less than one order of magnitude (see Figure 1a).

Overall, IC(0)-PCG using exact level-scheduled triangular solves implemented by cuSPARSE requires 660 seconds compared to 165 seconds when 3 Jacobi sweeps are used, a speedup of a factor of 4 . We note that this runtime does not include the preconditioner setup time, which can be large for the exact triangular solves as generating the level scheduling data structures is generally a sequential process. 
Figure 2 shows the corresponding results for the matrix Geo_1438. The behavior

\subsection{Increasing the factorization accuracy}

In Figure 5 we analyze the relation between the optimal number of scalar Jacobi sweeps and the incomplete Cholesky preconditioner with different levels of fill-in for 350 the thermal_1 matrix. The PCG iteration counts in Figure 5a indicate that the number of Jacobi sweeps necessary to match using exact triangular solves (solid lines) increases with the level of fill-in. Figure 5b reveals that PCG using scalar Jacobi sweeps for the preconditioner application is faster in all cases than PCG based on exact triangular solves. Figure 5c suggests that the optimal number of Jacobi sweeps (giving the lowest consistent with the idea that one should try to solve more accurately with a factorization that is more accurate. 


\subsection{Comprehensive results for Jacobi and block Jacobi iterations}

This section presents results for a large number of test problems. With a few exceptions, we chose all the real symmetric positive definite matrices from the SuiteSparse Matrix Collection that are of order at least 1000 and that are not diagonal. Three matrices from the af_shell set were discarded because of their similarity to af_shell7, which was retained. The Andrews matrix was removed because it uses an integer data type for matrix values. The audikw_1 and Flan_1565 matrices were also removed as the IC(1) preconditioner for these matrices, even after RCM ordering, could not fit in GPU memory. We also discarded three matrices, bloweybq, LFAT5000, and LF10000, whose condition numbers reported at the SuiteSparse Matrix Collection website exceed $10^{17}$. This gives us a set of 161 matrices. From this set, we chose two subsets: those that can be solved by IC(0)-PCG (using exact triangular solves) within 3000 iterations

370 and those that can be solved in the same way using IC(1)-PCG. This resulted in a set of 69 matrices for IC(0)-PCG and a set of 78 matrices for IC(1)-PCG. We list the matrices of the two sets along with some key characteristics in the Appendix.

Our goal is to find what fraction of these test sets can be solved using IC $(k)$-PCG when the exact triangular solves are replaced by Jacobi and block Jacobi iterations. We allow up to 20 Jacobi or block Jacobi sweeps for each triangular solve. For block Jacobi, we use supervariable amalgamation with a maximum block size of $m=12$. This was chosen because many PDE systems have 3, 4, or 6 degrees of freedom per node.

Table 1 summarizes our findings. In the case of $\mathrm{IC}(0), 84 \%$ of the problems that 380 can be solved by IC(0)-PCG can also be solved when the exact triangular solves are replaced by Jacobi iterations. The fraction increases to $92 \%$ when block Jacobi is used. For IC(1), the fractions are $74 \%$ and $94 \%$, respectively. These results show that for a significant proportion of our test problems, the exact triangular solves can be replaced by iterative solves. We note, however, that level-based incomplete Cholesky factoriza385 tions may not provide the best preconditioner among all possible preconditioners for all of the test problems; our results only show the potential efficacy of iterative triangular solves if a level-based incomplete Cholesky factorizations is used as the preconditioner.

Table 1: Number of problems that can be solved when exact triangular solves in $\operatorname{IC}(k)$-PCG $(k=0,1)$ are replaced by Jacobi and block Jacobi triangular solves.

\begin{tabular}{lll} 
& IC $(0)$ & IC(1) \\
\hline Num. solved using exact triangular solves & 69 & 78 \\
Num. solved using Jacobi triangular solves & $58(84 \%)$ & $58(74 \%)$ \\
Num. solved using block Jacobi triangular solves & $65(92 \%)$ & $73(94 \%)$ \\
\hline
\end{tabular}

For the problems that are successfully solved using Jacobi and block Jacobi, Figure 6 shows how many Jacobi and block Jacobi sweeps, respectively, are needed to minimize the IC $(k)$-PCG iteration run time. The figures are histograms showing the frequency of the number of sweeps. In all cases, there is a clustering of the optimal number of sweeps around 3, and for most of the problems the optimal number of sweeps is fewer than 10 . 
Figure 7 shows the speedup of the PCG run iteration time when using Jacobi and compared to using exact level-scheduled triangular solves implemented within cuSPARSE. The plots show that the speedup can exceed $10 \times$ and that only a small number of problems suffer a slow down. The results also show that compared to scalar Jacobi, block Jacobi results in fewer problems experiencing a slow down.

${ }_{400}$ We observe from Table 1 that using Jacobi iterations for IC(1)-PCG solves results in a smaller percentage of problems being solved compared to using Jacobi iterations for IC(0)-PCG. This suggests that the IC(1) incomplete factors are less diagonally dominant than the $\mathrm{IC}(0)$ factors. On the other hand, we found a similar percentage of problems that were solved when block Jacobi iterations are used for IC(0)-PCG and for IC(1)-PCG.

To investigate these issues, we compute the off-diagonal dominance of the IC(0) and IC(1) incomplete factors. We define the off-diagonal dominance of an $n \times n$ matrix $A$ as

$$
\frac{1}{n} \sum_{i}^{n} \sum_{j \neq i}^{n} \frac{\left|a_{i j}\right|}{\left|a_{i i}\right|} .
$$

Likewise, we define block off-diagonal dominance of a matrix $A$ with $k \times k$ blocking by

$$
\frac{1}{k} \sum_{i}^{k} \sum_{j \neq i}^{k} \frac{\left\|A_{i j}\right\|}{\left\|A_{i i}^{-1}\right\|^{-1}},
$$

where $A_{i j}$ denotes $(i, j)$-th block of the matrix. This definition follows the definition of block diagonal dominance of Feingold and Varga [35]. For convenience, we use the Frobenius norm in the above definition.

Figure 8 plots these two quantities for the 65 matrices in our test set that have both IC(0) and IC(1) factors. The results show that for nearly every matrix, the (block) 415 off-diagonal dominance of the IC(1) factor is either the same or higher than for the $\mathrm{IC}(0)$ factor. This suggests that Jacobi iterations will become less effective as more accurate incomplete factorizations are used. However, the numerical results we have already presented indicate that using block Jacobi with IC(1) we are able to solve a large fraction of the test problems where IC(1) was successful with exact solves.

\subsection{Results with priority blocking}

The blocking technique used may have a large influence on the results of block Jacobi iteration. For simplicity, we used supervariable amalgamation in our experiments. In this section, however, we briefly show that more delicate blocking techniques may be effective. Our motivation is not to advocate a single blocking method, but to emphasize that a user should carefully select or design a blocking method that is suitable for the problem.

From the results for IC(0)-PCG in Table 1, there are four test examples that could not be solved when using block Jacobi solves with supervariable amalgamation but were solved using direct triangular solves. These examples are problems cfd1, crankseg_1, 
Table 2: For IC(0), results using exact triangular solves (cuSPARSE) for problems using three different orderings: RCM, PB, and PBD. The table shows the number of IC(0)-PCG iterations, the iteration run time, and the number of levels in the level scheduling.

\begin{tabular}{l|rrr|rrr|rrr}
\hline & \multicolumn{3}{|c|}{ IC(0)-PCG iterations } & \multicolumn{3}{|c}{ Run time } & \multicolumn{3}{c}{ Number of levels } \\
& RCM & PB & PBD & RCM & PB & PBD & RCM & PB & PBD \\
\hline cfd1 & 445 & 496 & 537 & 24.8 & 15.7 & 32.4 & 2831 & 1249 & 2903 \\
crankseg_1 & 88 & 190 & 163 & 23.3 & 99.8 & 96.7 & 6841 & 13594 & 16086 \\
Emilia_923 & 305 & 604 & 1346 & 226.1 & 99.7 & 269.8 & 33495 & 3749 & 4938 \\
\hline
\end{tabular}

crankseg_2, and Emilia_923. We now show that these can be solved using the two priority blocking methods mentioned earlier. Note that detailed results for crankseg_2 are not shown, because of its similarity to crankseg_1.

Recall that once the blocks are identified by a priority blocking algorithm, an RCM ordering is applied to the condensed graph to determine the final ordering of the matrix.

${ }_{435}$ This ordering generally differs from an RCM ordering of the original matrix. We refer to the orderings resulting from the priority blocking and priority blocking with dynamic edge weight algorithms as PB and PBD orderings, respectively. We first compare the effect of the RCM, PB, and PBD orderings on convergence and parallel performance using IC(0)-PCG with exact triangular solves. For the PB and PBD orderings, the blockings are determined using a maximum block size of 12 , the same value as was used earlier for supervariable amalgamation. Results are given in Table 2. In addition to the IC(0)-PCG iteration counts and the iteration run time, the number of levels in the level scheduling for the exact triangular solves is reported because this affects how efficiently the exact triangular solves are parallelized. We observe that for our three test 445 matrices, the PB and PBD orderings generally lead to increased iteration counts, confirming that RCM is a good ordering for incomplete Cholesky factorizations. However, we also observe the anomaly that the PB and PBD orderings can reduce the run times. Because of the locations of large nonzeros in the matrix, PB and PBD can (but not always) result in an ordering that reduces the number of levels, meaning that the level450 scheduled triangular solves can be more efficiently parallelized, leading to a decrease in the iteration run time.

We next use the PB and PBD orderings for IC(0)-PCG with block Jacobi solves. We also test orderings generated from METIS partitionings of the matrices using edge weights (with average block size 12). As with the PB and PBD orderings, the METIS 455 ordering is obtained by applying RCM to a condensed graph. In Figure 9 (bottom row) we present results for the METIS ordering for problems cfd1 and crankseg_1; the IC $(0)$ factorization broke down for problem Emilia_923 with the METIS ordering so is omitted. Note that we also tested TPABLO on these matrices using a maximum block size of 12 and a range of parameter values, including the default. However, the block Jacobi solves converged slowly and did not provide effective preconditioners.

Figure 9 shows the IC(0)-PCG iteration counts (left column) and the iteration run time (right column) as the number of block Jacobi sweeps is varied. Although the block Jacobi triangular solves were not effective when supervariable amalgamation was used for these matrices, we observe that after only a small number of block Jacobi sweeps 
Table 3: The optimal number of block Jacobi sweeps and the corresponding number of IC(0)-PCG iterations and iteration time for the PB and PBD orderings.

\begin{tabular}{l|crr|crrr}
\hline & \multicolumn{3}{|c|}{ PB } & \multicolumn{3}{c}{ PBD } \\
& sweeps & iters & time & sweeps & iters & time \\
\hline cfd1 & 1 & 1298 & 4.0 & 4 & 651 & 3.3 \\
crankseg_1 & 6 & 209 & 4.4 & 7 & 171 & 3.9 \\
Emilia_923 & 7 & 625 & 72.8 & 5 & 1392 & 139.2 \\
\hline
\end{tabular}

(fewer than 10), the number of IC(0)-PCG iterations is almost the same as for exact triangular solves. The METIS orderings give IC(0)-PCG iteration run times that are worse than those for the PB and PBD orderings.

Table 3 shows the optimal number of block Jacobi sweeps for the PB and PBD orderings to minimize the iteration run time. The corresponding number of PCG iterations and the run time are also reported. The timings in this table can be compared to those in Table 2. We observe that for each system, the best run times for block Jacobi sweeps are always faster than using exact triangular solves. If we compare block Jacobi solves using the $\mathrm{PB}$ ordering to exact triangular solves using RCM, the run time speedups are 6.2, 5.3, and 3.1 for our three test matrices.

475 From the results for IC(1) in Table 1, there are five problems that could not be solved when using block Jacobi with supervariable amalgamation. These problems are nd3k, crankseg_1, crankseg_2, m_t1, and Emilia_923. Once again, we try to solve these problems with the two priority blocking methods. In this case, we are able to solve all the problems except $m_{-} t$. Tables 4 and 5 report results for IC(1)-PCG analogous to those for IC(0)-PCG shown in Tables 2 and 3, respectively. (Again, we only show results for crankseg_1 and not for crankseg_2.) We see that IC(1)-PCG with iterative triangular solves is faster than using exact triangular solves.

Table 4: For IC(1), results using exact triangular solves (cuSPARSE) for problems using three different orderings: RCM, PB, and PBD. The table shows the number of IC(1)-PCG iterations, the iteration run time, and the number of levels in the level scheduling.

\begin{tabular}{l|rrr|rrr|rrr}
\hline & \multicolumn{4}{|c|}{ PCG iterations } & \multicolumn{3}{c|}{ Time } & \multicolumn{3}{c}{ Num. levels } \\
& RCM & PB & PBD & RCM & PB & PBD & RCM & PB & PBD \\
\hline nd3k & 51 & 63 & 58 & 38.1 & 50.4 & 44.7 & 7716 & 7564 & 7052 \\
crankseg_1 & 40 & 59 & 49 & 39.2 & 177.9 & 142.6 & 15856 & 33357 & 32864 \\
Emilia_923 & 102 & 496 & 1121 & 149.3 & 359.8 & 1220.8 & 60264 & 17913 & 31071 \\
\hline
\end{tabular}

The system with matrix $m_{-} t 1$ could not be solved with IC(1)-PCG using either Jacobi or block Jacobi sweeps. We note that $\mathrm{m}_{-} \mathrm{t} 1$ has large off-diagonal entries, essentially as large as the diagonal values themselves. When a matrix has a full subdiagonal of large nonzero values, for example, there is no blocking that can prevent some of these large entries from being outside a block diagonal structure. 
Table 5: The optimal number of block Jacobi sweeps and the corresponding number of IC(1)-PCG iterations and iteration time for PB and PBD ordering.

\begin{tabular}{l|crr|crr}
\hline & \multicolumn{3}{|c|}{ PB } & \multicolumn{3}{c}{ PBD } \\
& sweeps & iters & time & sweeps & iters & time \\
\hline nd3k & 22 & 69 & 1.8 & 23 & 60 & 1.4 \\
crankseg_1 & 9 & 61 & 3.0 & 9 & 54 & 2.6 \\
Emilia_923 & 8 & 496 & 99.0 & 5 & 1181 & 167.3 \\
\hline
\end{tabular}

\section{Conclusions}

The main goal of this paper is to understand the applicability of Jacobi and block Jacobi iterations for solving the sparse triangular systems arising from incomplete Cholesky preconditioning. As always, artificial problems can be constructed to defeat the Jacobi approach. However, we find that for a diverse set of realistic symmetric positive definite test problems using IC(0)-PCG and IC(1)-PCG, Jacobi iterations are effective for a large proportion of the problems. We also showed that by using block Jacobi, robustness could be enhanced. Even better results for some problems could be obtained by using blocking techniques that use matrix values (e.g., priority blocking).

We note that these results do depend on the parallelism of the computing architecture. The use of (block) Jacobi is targeted for high parallelism cases, like GPUs and manycore CPUs. With lower amounts of parallelism, there are two main effects: (1) the 500 advantage of using (block) Jacobi over exact solves inside PCG is smaller or may not be effective, and (2) it is possible that the PCG solve time as a function of the number of sweeps might only increase and not decrease, i.e., more sweeps always increases the PCG solve time because the (block) Jacobi triangular solves are not computed fast enough for there to be an advantage to using multiple sweeps.

This paper did not address how to choose the optimal number of Jacobi sweeps to use in the PCG solver. A fixed number of sweeps is desirable, as the preconditioner is then a fixed operator and a "flexible" solver is not needed. However, in practice, the number of sweeps could be adjusted dynamically and the iterations restarted based on the convergence of PCG. If a flexible solver is available, e.g, FGMRES [36] or flexible conjugate gradients $[37,38]$, then a different number of Jacobi sweeps based on the residual norm reduction for each approximate triangular solve could be used.

A caveat when reading the results of this paper is that no preconditioner is best for all problems, as the choice of preconditioner must be matched to the difficulty (e.g., conditioning) of the problem. Thus in this paper we do not imply that incomplete ${ }_{515}$ factorization preconditioning combined with Jacobi iterative triangular solves is the best method to use for all problems. Instead, if incomplete factorization is deemed to be effective for a given problem compared to other preconditioners, then it is likely that Jacobi triangular solves will yield a speedup over traditional triangular solves on highly parallel computer architectures. 


\section{Acknowledgments}

This research was supported by the Department of Energy, Office of Science, Office of Advanced Scientific Computing Research, Applied Mathematics program under Award Numbers DE-SC-0016564 and DE-SC-0016513. This research was also supported by EPSRC grant EP/I013067/1. H. Anzt was partially supported by the "Impuls und Vernetzungsfond of the Helmholtz Association" under grant VH-NG-1241.

\section{References}

[1] E. C. Anderson, Y. Saad, Solving sparse triangular systems on parallel computers, International J. of High Speed Computing 1 (1989) 73-96.

[2] S. W. Hammond, R. Schreiber, Efficient ICCG on a shared memory multiprocessor, International J. High Speed Computing 4 (1992) 1-21.

[3] J. H. Saltz, Aggregation methods for solving sparse triangular systems on multiprocessors, SIAM J. on Scientific and Statistical Computing 11 (1) (1990) 123144.

[4] M. M. Wolf, M. A. Heroux, E. G. Boman, Factors impacting performance of multithreaded sparse triangular solve, High Performance Computing for Computational Science - VECPAR 20106449 (2010) 32-44.

[5] R. Li, Y. Saad, GPU-accelerated preconditioned iterative linear solvers, The Journal of Supercomputing 63 (2) (2013) 443-466.

[6] H. Anzt, E. Chow, J. Dongarra, Iterative sparse triangular solves for preconditioning, Lecture Notes in Computer Science 9233 (2015) 650-661.

[7] E. Chow, A. Patel, Fine-grained parallel incomplete LU factorization, SIAM J. on Scientific Computing 37 (2) (2015) C169-C193.

[8] M. Benzi, M. Tůma, A comparative study of sparse approximate inverse preconditioners, Applied Numerical Mathematics 30 (1999) 305-340.

[9] A. C. N. van Duin, Scalable parallel preconditioning with the sparse approximate inverse of triangular matrices, SIAM J. on Matrix Analysis and Applications 20 (4) (1999) 987-1006.

[10] F. L. Alvarado, R. Schreiber, Optimal parallel solution of sparse triangular systems, SIAM J. on Scientific Computing 14 (2) (1993) 446-460.

[11] A. Pothen, F. L. Alvarado, A fast reordering algorithm for parallel sparse triangular solution, SIAM J. on Scientific Computing 13 (2) (1992) 645-653.

[12] H. A. Van der Vorst, A vectorizable variant of some ICCG methods, SIAM J. on Scientific Computing 3 (1982) 350-356. 
[13] H. Anzt, T. K. Huckle, J. Bräckle, J. Dongarra, Incomplete sparse approximate inverses for parallel preconditioning, Parallel Computing 71 (Supplement C) (2018) $1-22$.

[14] M. T. Jones, P. E. Plassmann, Scalable iterative solution of sparse linear-systems, Parallel Computing 20 (5) (1994) 753-773.

[15] E. H. Cuthill, J. McKee, Reducing the bandwidth of sparse symmetric matrices, in: Proceedings $24^{\text {th }}$ National Conference of the ACM, ACM Press, 1969, pp. $157-172$.

[16] S. W. Sloan, An algorithm for profile and wavefront reduction of sparse matrices, International J. of Numerical Methods in Engineering 23 (1986) 239-251.

[17] M. Benzi, W. D. Joubert, G. Mateescu, Numerical experiments with parallel orderings for ILU preconditioners, Electronic Transactions on Numerical Analysis 8 (1999) 88-114.

[18] S. Doi, On parallelism and convergence of incomplete LU factorizations, Applied Numerical Mathematics 7 (5) (1991) 417-436.

[19] S. Doi, T. Washio, Ordering strategies and related techniques to overcome the trade-off between parallelism and convergence in incomplete factorizations, Parallel Computing 25 (13-14) (1999) 1995-2014.

[20] I. S. Duff, G. A. Meurant, The effect of ordering on preconditioned conjugate gradients, BIT Numerical Mathematics 29 (4) (1989) 635-657.

[21] H. C. Elman, E. Agrón, Ordering techniques for the preconditioned conjugategradient method on parallel computers, Computer Physics Communications 53 (1-3) (1989) 253-269.

[22] E. L. Poole, J. M. Ortega, Multicolor ICCG methods for vector computers, SIAM J. on Numerical Analysis 24 (6) (1987) 1394-1417.

[23] E. Chow, Y. Saad, Experimental study of ILU preconditioners for indefinite matrices, Journal of Computational and Applied Mathematics 86 (2) (1997) 387-414.

[24] C. Ashcraft, Compressed graphs and the minimum degree algorithm, SIAM Journal on Scientific Computing 16 (6) (1995) 1404-1411.

[25] Y. Saad, Finding exact and approximate block structures for ILU preconditioning, SIAM Journal on Scientific Computing 24 (4) (2003) 1107-1123.

[26] J. D. Hogg, J. A. Scott, A modern analyse phase for sparse tree-based direct methods, Numerical Linear Algebra with Applications 20 (2013) 397-412.

[27] G. Karypis, V. Kumar, A fast and high quality multilevel scheme for partitioning irregular graphs, SIAM Journal on Scientific Computing 20 (1) (1998) 359-392. 
[28] D. Fritzsche, A. Frommer, D. B. Szyld, Extensions of certain graph-based algorithms for preconditioning, SIAM J. on Scientific Computing 29 (2007) 21442161.

[29] J. ONeil, D. B. Szyld, A block ordering method for sparse matrices, SIAM Journal on Scientific and Statistical Computing 11 (5) (1990) 811-823.

[30] I. S. Duff, K. Kaya, Preconditioners based on strong subgraphs, Electronic Transactions on Numerical Analysis 40 (2013) 225-248.

[31] R. E. Tarjan, An improved algorithm for hierarchical clustering using strong components, Information Processing Letters 17 (1) (1983) 37-41.

[32] T. A. Davis, Y. Hu, The University of Florida sparse matrix collection, ACM Transactions on Mathematical Software 38, article 1, 25 pages.

600 [33] Innovative Computing Lab, Software distribution of MAGMA version 2.2.0, http://icl.cs.utk. edu/magma/ (2016).

[34] H. Anzt, J. Dongarra, G. Flegar, E. S. Quintana-Ortí, Variable-size batched Gauss-Jordan elimination for block-Jacobi preconditioning on graphics processors, Parallel Computing, 2018.

605 [35] D. G. Feingold, R. S. Varga, Block diagonally dominant matrices and generalizations of the Gerschgorin circle theorem., Pacific Journal of Mathematics 12 (4) (1962) 1241-1250.

[36] Y. Saad, A flexible inner-outer preconditioned GMRES algorithm, SIAM J. on Scientific and Statistical Computing 14 (1993) 461-469.

610 [37] O. Axelsson, Iterative Solution Methods, Cambridge University Press, Cambridge, 1994.

[38] Y. Notay, Flexible conjugate gradients, SIAM Journal on Scientific Computing 22 (4) (2000) 1444-1460. 


\section{Appendix}

615 Al: Problems for the $I C(0)$ analysis

\begin{tabular}{|c|c|c|c|c|c|}
\hline Name & \#rows & \#nonzeros & Name & \#rows & \#nonzeros \\
\hline e1138_bus & 1138 & 4054 & wathen 100 & 30401 & 471601 \\
\hline Chem 97ZtZ & 2541 & 7361 & gridgena & 48962 & 512084 \\
\hline bcsstk 08 & 1074 & 12960 & apachel & 80800 & 542184 \\
\hline mhd3200b & 3200 & 18316 & wathen 120 & 36441 & 565761 \\
\hline bcsstm 12 & 1473 & 19659 & thermall & 82654 & 574458 \\
\hline mhd4800b & 4800 & 27520 & crystm 03 & 24696 & 583770 \\
\hline plbuckle & 1282 & 30644 & finan512 & 74752 & 596992 \\
\hline bcsstk 27 & 1224 & 56126 & thermomech_TC & 102158 & 711558 \\
\hline nasa2146 & 2146 & 72250 & thermomech_TK & 102158 & 711558 \\
\hline fv1 & 9604 & 85264 & Pres_Poisson & 14822 & 715804 \\
\hline fv2 & 9801 & 87025 & G2_circuit & 150102 & 726674 \\
\hline crystm01 & 4875 & 105339 & bundle 1 & 10581 & 770811 \\
\hline bodyy 4 & 17546 & 121550 & Dubcova2 & 65025 & 1030225 \\
\hline aft01 & 8205 & 125567 & thermomech_dM & 204316 & 1423116 \\
\hline bodyy 5 & 18589 & 128853 & t2cubes_sphere & 101492 & 1647264 \\
\hline bodyy 6 & 19366 & 134208 & qa8fm & 66127 & 1660579 \\
\hline ted_B & 10605 & 144579 & $\operatorname{cfd} 1$ & 70656 & 1825580 \\
\hline ted_B_unscaled & 10605 & 144579 & Dubcova3 & 146689 & 3636643 \\
\hline Muu & 7102 & 170134 & parabolic_fem & 525825 & 3674625 \\
\hline t2dah_e & 11445 & 176117 & offshore & 259789 & 4242673 \\
\hline obstclae & 40000 & 197608 & pdb1HYS & 36417 & 4344765 \\
\hline torsion 1 & 40000 & 197608 & apache2 & 715176 & 4817870 \\
\hline jnlbrng 1 & 40000 & 199200 & tmt_sym & 726713 & 5080961 \\
\hline minsurfo & 40806 & 203622 & G3_circuit & 1585478 & 7660826 \\
\hline $\mathrm{s} 1 \mathrm{rmt} 3 \mathrm{~m} 1$ & 5489 & 217651 & thermal2 & 1228045 & 8580313 \\
\hline $\mathrm{s} 2 \mathrm{rmt} 3 \mathrm{~m} 1$ & 5489 & 217681 & crankseg_1 & 52804 & 10614210 \\
\hline Dubcoval & 16129 & 253009 & crankseg_2 & 63838 & 14148858 \\
\hline $\mathrm{s} 1 \mathrm{rmq} 4 \mathrm{~m} 1$ & 5489 & 262411 & af_shell7 & 504855 & 17579155 \\
\hline $\mathrm{s} 2 \mathrm{rmg} 4 \mathrm{~m} 1$ & 5489 & 263351 & bundle_adj & 513351 & 20207907 \\
\hline bcsstk16 & 4884 & 290378 & Emilia_923 & 923136 & 40373538 \\
\hline crystm02 & $\begin{array}{r}4004 \\
13965\end{array}$ & 322905 & Hook_1498 & 1498023 & 59374451 \\
\hline shallow_water1 & 81920 & 327680 & Geo_1438 & 1437960 & 60236322 \\
\hline shallow_water2 & 81920 & 327680 & Trefethen_2000 & 2000 & 41906 \\
\hline Kuu & 7102 & 340200 & Trefethen_20000 & 20000 & 554466 \\
\hline gyro_m & 17361 & 340431 & & & \\
\hline
\end{tabular}

Table 6: Table listing the matrices along with key characteristics that are included in the IC(0) tests. 
A2: Problems for the IC(1) analysis

\begin{tabular}{|c|c|c|c|c|c|}
\hline Name & \#rows & \#nonzeros & Name & \#rows & \#nonzeros \\
\hline e1138_bus & 1138 & 4054 & crystm02 & 13965 & 322905 \\
\hline Chem97ZtZ & 2541 & 7361 & shallow_water1 & 81920 & 327680 \\
\hline bcsstk08 & 1074 & 12960 & shallow_water2 & 81920 & 327680 \\
\hline mhd3200b & 3200 & 18316 & Kuu & 7102 & 340200 \\
\hline bcsstm 12 & 1473 & 19659 & gyro_m & 17361 & 340431 \\
\hline bcsstk10 & 1086 & 22070 & wathen 100 & 30401 & 471601 \\
\hline $\mathrm{msc} 01050$ & 1050 & 26198 & gridgena & 48962 & 512084 \\
\hline $\mathrm{mhd} 4800 \mathrm{~b}$ & 4800 & 27520 & apache1 & 80800 & 542184 \\
\hline plbuckle & 1282 & 30644 & wathen 120 & 36441 & 565761 \\
\hline bcsstk 27 & 1224 & 56126 & thermal1 & 82654 & 574458 \\
\hline bcsstk14 & 1806 & 63454 & crystm03 & 24696 & 583770 \\
\hline nasa2146 & 2146 & 72250 & finan 512 & 74752 & 596992 \\
\hline fv1 & 9604 & 85264 & cbuckle & 13681 & 676515 \\
\hline fv2 & 9801 & 87025 & thermomech_TC & 102158 & 711558 \\
\hline $\mathrm{msc} 04515$ & 4515 & 97707 & thermomech_TK & 102158 & 711558 \\
\hline crystm01 & 4875 & 105339 & Pres_Poisson & 14822 & 715804 \\
\hline bcsstk 15 & 3948 & 117816 & G2_circuit & 150102 & 726674 \\
\hline bodyy4 & 17546 & 121550 & bundle1 & 10581 & 770811 \\
\hline aft01 & 8205 & 125567 & Dubcova2 & 65025 & 1030225 \\
\hline bodyy 5 & 18589 & 128853 & thermomech_dM & 204316 & 1423116 \\
\hline bodyy 6 & 19366 & 134208 & t2cubes_sphere & 101492 & 1647264 \\
\hline ted_B & 10605 & 144579 & qa8fm & 66127 & 1660579 \\
\hline ted_B_unscaled & 10605 & 144579 & $\mathrm{nd} 3 \mathrm{k}$ & 9000 & 3279690 \\
\hline bcsstk24 & 3562 & 159910 & Dubcova3 & 146689 & 3636643 \\
\hline Muu & 7102 & 170134 & parabolic_fem & 525825 & 3674625 \\
\hline t2dah_e & 11445 & 176117 & cant & 62451 & 4007383 \\
\hline obstclae & 40000 & 197608 & pdb1HYS & 36417 & 4344765 \\
\hline torsion 1 & 40000 & 197608 & apache2 & 715176 & 4817870 \\
\hline jnlbrng1 & 40000 & 199200 & ecology 2 & 999999 & 4995991 \\
\hline minsurfo & 40806 & 203622 & tmt_sym & 726713 & 5080961 \\
\hline $\mathrm{s} 3 \mathrm{rmt} 3 \mathrm{~m} 3$ & 5357 & 207123 & G3_circuit & 1585478 & 7660826 \\
\hline $\mathrm{s} 1 \mathrm{rmt} 3 \mathrm{~m} 1$ & 5489 & 217651 & thermal2 & 1228045 & 8580313 \\
\hline $\mathrm{s} 3 \mathrm{rmt} 3 \mathrm{~m} 1$ & 5489 & 217669 & $\mathrm{~m}_{\mathrm{t}} \mathrm{t}$ & 97578 & 9753570 \\
\hline $\mathrm{s} 2 \mathrm{rmt} 3 \mathrm{~m} 1$ & 5489 & 217681 & crankseg_1 & 52804 & 10614210 \\
\hline Dubcova1 & 16129 & 253009 & crankseg_2 & 63838 & 14148858 \\
\hline $\mathrm{s} 1 \mathrm{rmq} 4 \mathrm{~m} 1$ & 5489 & 262411 & af_shell7 & 504855 & 17579155 \\
\hline $\mathrm{s} 3 \mathrm{rmq} 4 \mathrm{~m} 1$ & 5489 & 262943 & Emilia_923 & 923136 & 40373538 \\
\hline $\mathrm{s} 2 \mathrm{rmq} 4 \mathrm{~m} 1$ & 5489 & 263351 & Hook_1498 & 1498023 & 59374451 \\
\hline bcsstk16 & 4884 & 290378 & Geo_1438 & 1437960 & 60236322 \\
\hline
\end{tabular}

Table 7: Table listing the matrices along with key characteristics that are included in the IC(1) tests. 


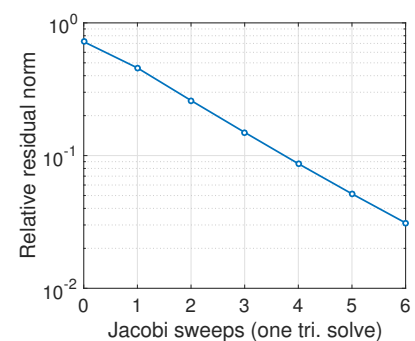

(a) Scalar Jacobi triangular solve convergence.

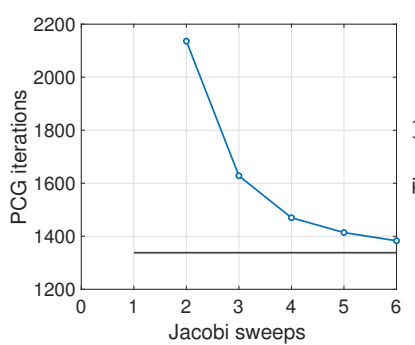

(b) IC(0)-PCG convergence.

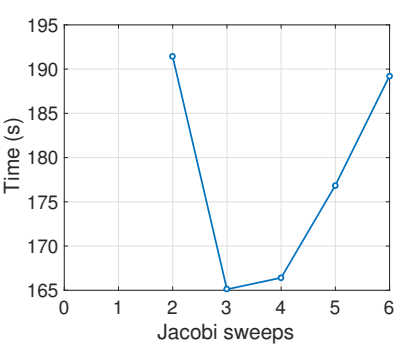

(c) IC(0)-PCG run time.

Figure 1: For Hook_1498, the effects of increasing the number of scalar Jacobi sweeps. Using exact triangular solves requires $1338 \mathrm{IC}(0)$-PCG iterations and 660 seconds.

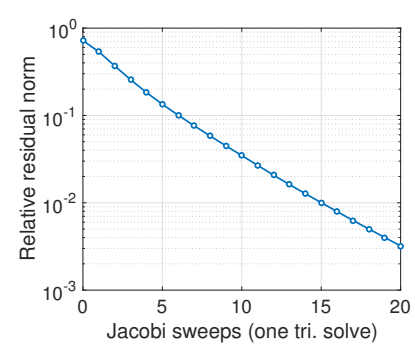

(a) Scalar Jacobi triangular solve convergence.

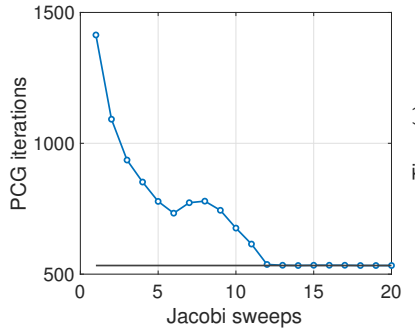

(b) IC(0)-PCG convergence.

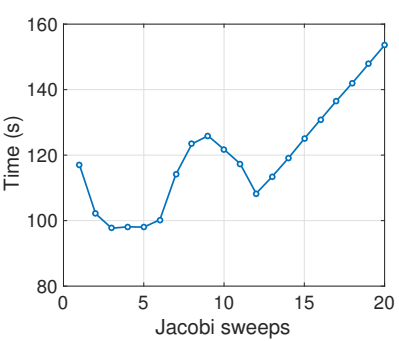

(c) $\mathrm{IC}(0)-\mathrm{PCG}$ run time.

Figure 2: For Geo_1438, the effects of increasing the number of scalar Jacobi sweeps. Using exact triangular solves requires $533 \mathrm{IC}(0)$-PCG iterations and 320 seconds.
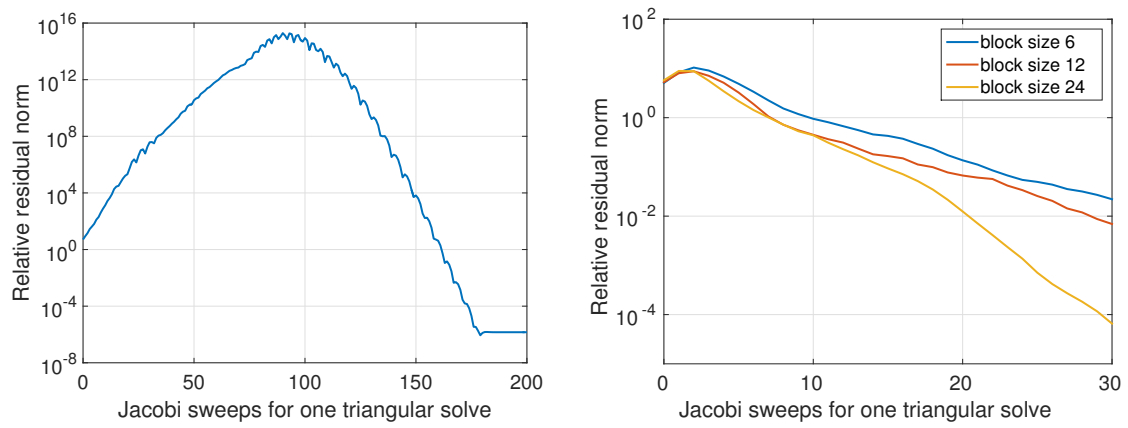

Figure 3: For bcsstk24, the relative residual norm for a triangular solve with the lower triangular IC(1) factor and a random right-hand side, without blocking (left) and with supervariable amalgamation (right). 

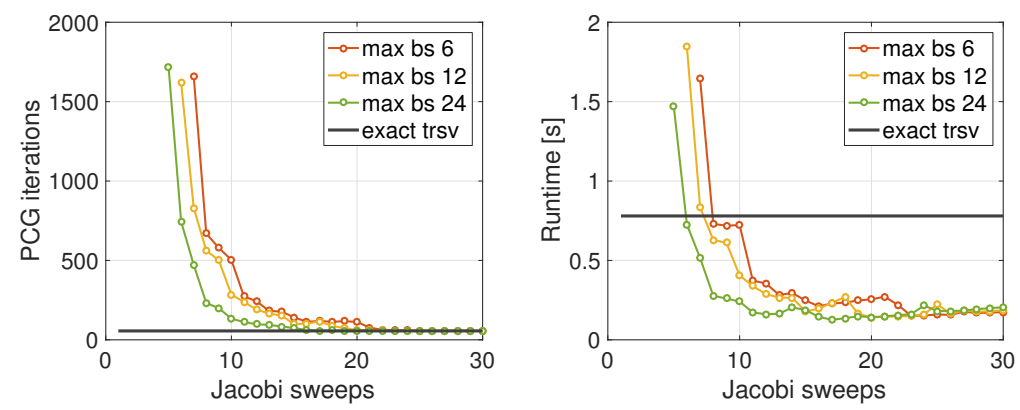

Figure 4: For bcsstk24, the effect of increasing the number of Jacobi sweeps on IC(1)-PCG iteration counts (left) and run times (right). Different maximum block sizes are used (max bs). The result of using exact triangular solves is also shown.

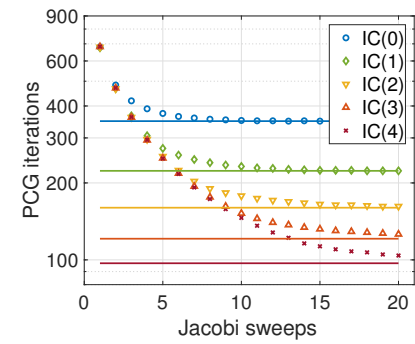

(a) PCG iterations using IC preconditioning with different levels of fill.

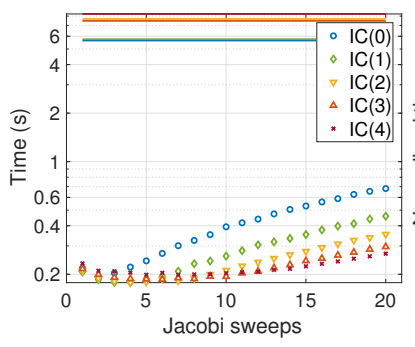

(b) PCG run time.

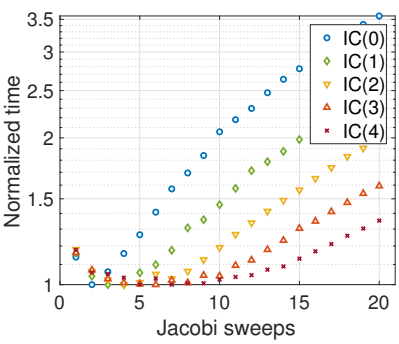

(c) PCG run time, normalized by the fastest run time for each IC level.

Figure 5: For thermal_1, the effects of increasing the number of scalar Jacobi sweeps. 


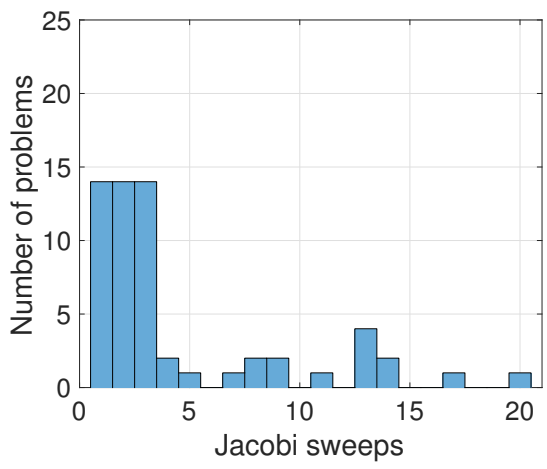

(a) $\mathrm{IC}(0)+$ scalar Jacobi.

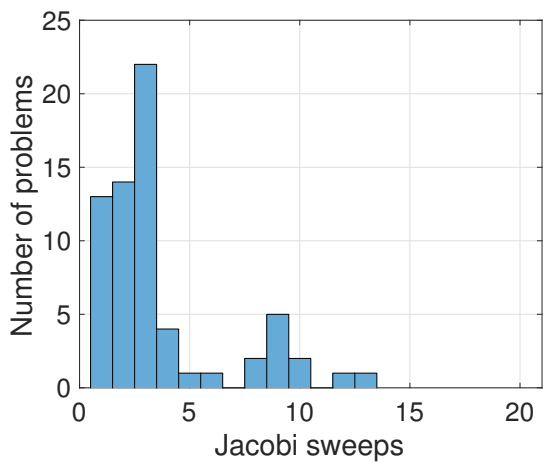

(c) $\mathrm{IC}(0)+$ block Jacobi.

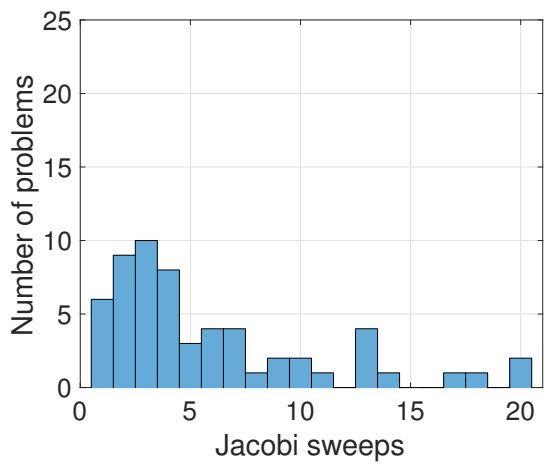

(b) IC(1) + scalar Jacobi.

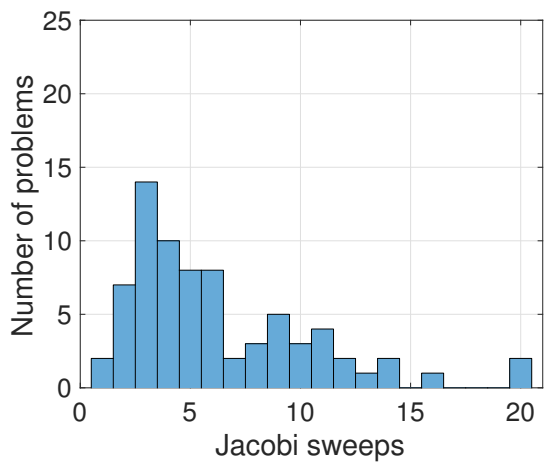

(d) IC(1) + block Jacobi.

Figure 6: Optimal number of sweeps to minimize PCG iteration run time. The block Jacobi results (bottom row) use supervariable amalgamation and a maximum block size of 12 . 

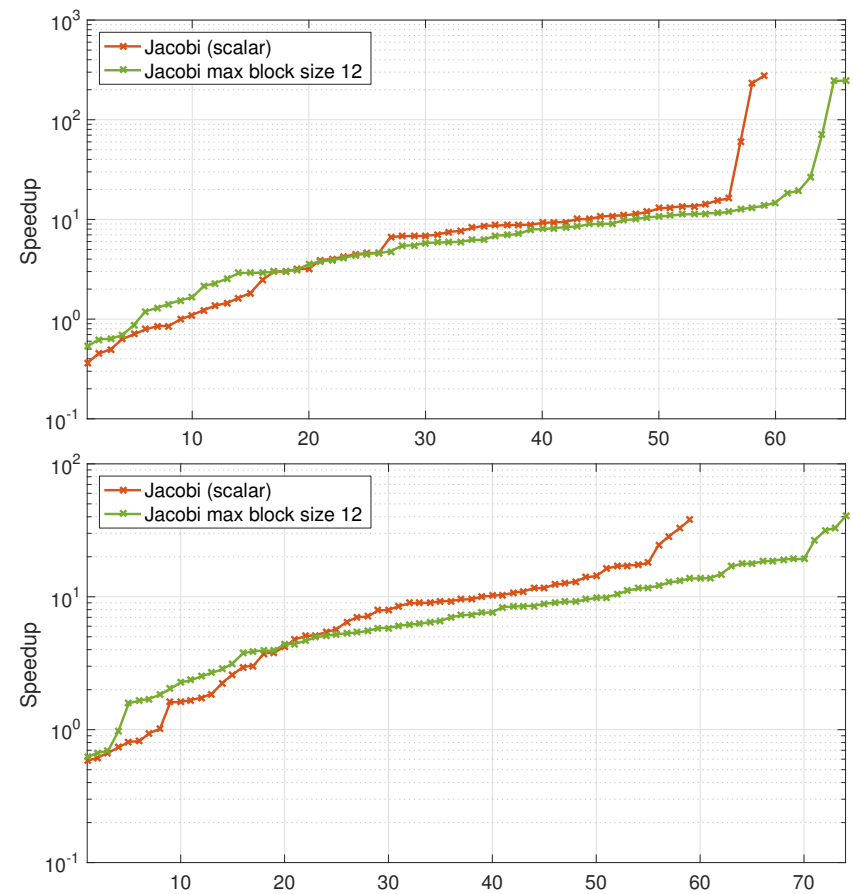

Figure 7: Speedup of the PCG iteration run time when using Jacobi and block Jacobi triangular solves compared to using exact triangular solves. Top: IC(0). Bottom: IC(1). Matrix problems on the $x$-axis are sorted by speedup, and are not the same for the different curves. Note that the curves have different lengths because different numbers of problems can be solved for each preconditioner configuration (block size and IC level). These speedups are for the same runs shown in the previous histograms. 

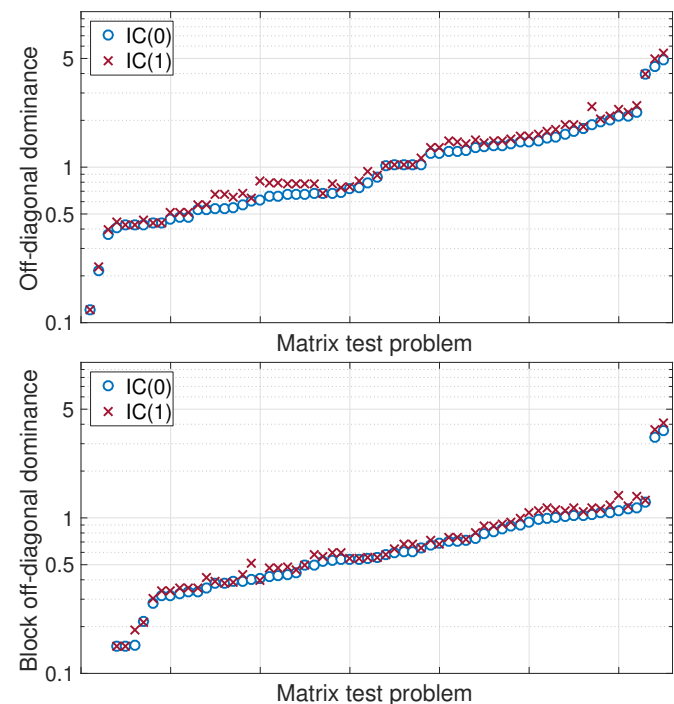

Figure 8: Off-diagonal dominance (top) and block off-diagonal dominance (bottom) for 65 test matrices, with matrices sorted by the value for the $\mathrm{IC}(0)$ factor. Smaller values means a matrix is more diagonally dominant. 
cfd1
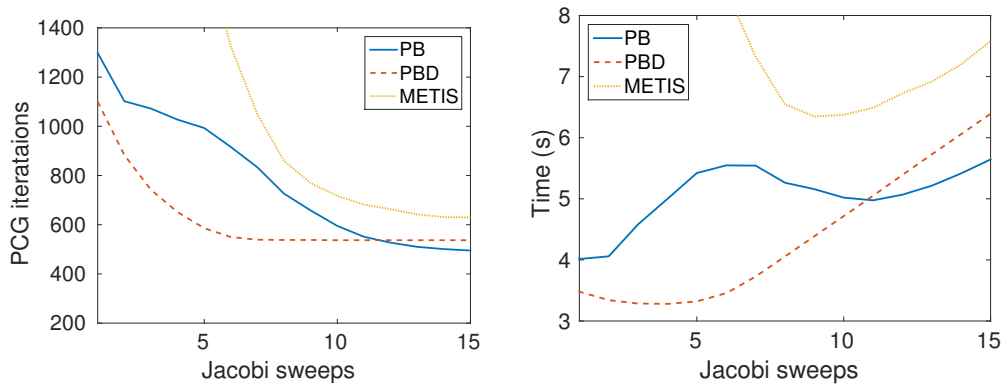

crankseg_1
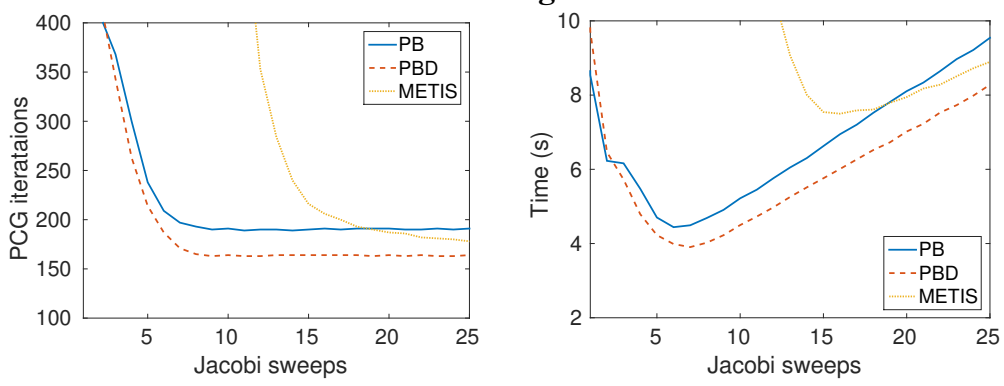

Emilia_923
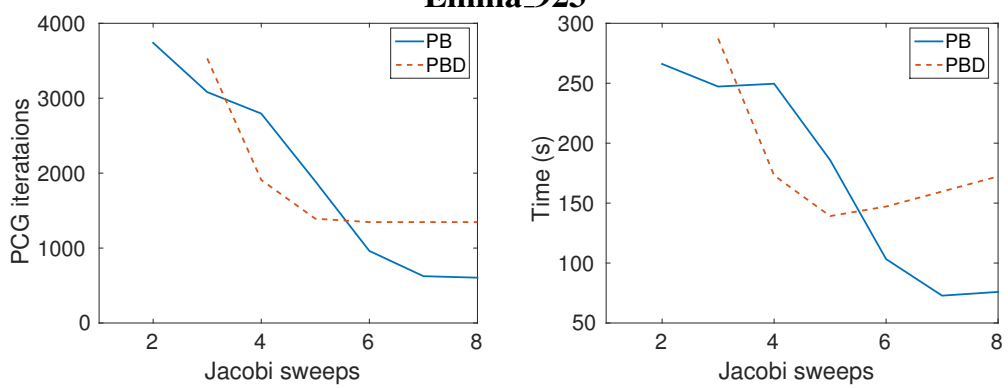

Figure 9: IC(0)-PCG iterations and iteration run time as a function of the number of block Jacobi sweeps, using the PB, PBD, and METIS orderings. 